\title{
ON UNSTEADY HYDROMAGNETIC FLOWS OF A DUSTY VISCOUS FLUID BETWEEN TWO OSCILLATING PLATES*
}

\author{
Lokenath Debnath \\ Department of Mathematics \\ University of Central Florida \\ Orlando, FL 32816, U.S.A. \\ and \\ A. K. Ghosh \\ Department of Mathematics \\ Jadavpur University \\ Calcutta - 700 032, India
}

\begin{abstract}
A study is made of the unsteady motion of an incompressible viscous conducting fluid with embedded small spherical particles bounded by two infinite rigid non-conducting plates. The operational method derives exact solutions for the fluid and the particle velocities and the wall shear stress. The quantitative evaluation of these results is considered when the two plates oscillate in phase but with different frequencies. The results are shown graphically for different values of the time period of oscillations of the plates which represent the cases: (i) the lower plate oscillates with time period less than the upper, (ii) both the plates oscillate with the same time period, (iii) the lower plate oscillates with time period greater than the upper. The magnetic field damps the fluid motion for all values of the time period of oscillations of the plates. When the time periods are small, i.e., when the plates oscillate with high frequency, the fluid motion is retarded by the particles. However, when the plates oscillate with larger time periods (smaller frequencies), the fluid velocity is increased by the presence of the particles at the early stage of the motion, and this effect persists until the equilibrium is reached when the particles exert their influence to resist the flow. The drag on the plate, which is evaluated numerically for the lower plate oscillating with large time period, depends on the ratio of the time periods of the oscillating plates. If the ratio of the time periods is not equal to unity, the drag on the plate, irrespective of the values of the magnetic field, oscillates with larger amplitude compared to its value when the ratio of the time periods is equal to unity. Further, for the ratio of the time periods less than or equal to unity and for any fixed values of the magnetic field, the drag increases by the presence of the particles after a time $t \approx 1.2$ which is the upper time limit for the non-equilibrium stress-value to exist. In a similar situation, a reverse effect, i.e., the decrease of the drag with increasing particle concentration, is found for the ratio of the time periods being greater than unity.
\end{abstract}

Keywords and Phrases: Unsteady Hydromagnetic Flows, Dusty Viscous Fluids, Two Phase Flows, and Laplace Transforms.

AMS (MOS) Subject Classification: 76VO5

\footnotetext{
${ }^{*}$ Received 12/88; Accepted 1/89
} 


\section{INTRODUCTION}

Using the model of Saffman [1], Ghosh and Debnath [2] recently made an initial value investigation of various aspects of hydromagnetic two-phase viscous rotating fluid flows bounded by an infinite rigid non-conducting plate.

The unsteady hydromagnetic flow in an electrically conducting viscous fluid with suspended small spherical particles bounded by two infinite non-conducting parallel plates in the presence of an external transverse magnetic field warrants investigation. The exact solutions for the fluid and the particle velocities are determined by the Laplace transform method. The shear stresses on the plates are then obtained in the closed form. These solutions are evaluated numerically for instances when both the plates oscillate in phase but with different frequencies. The velocity profiles and the shear stresses on the plates are graphically represented for different values of the flow parameters. An increase of the magnetic field diminishes the fluid velocity for all values of the frequency (or period) of the oscillations of the plates. The presence of particles retards the fluid motion except for small frequency values or for large values of the time period of plate oscillations. For these large values, the fluid velocity is increased at the beginning due to the presence of particles until the equilibrium state is attained when the particles resist the fluid motion. The shear stress on the plates increases with the increase in particle concentration after the nonequilibrium process of particle relaxation is over. For large values of the time period of plate oscillations, the magnetic field reduces the amplitude of drag-oscillation up to a certain point after which the drag-amplitude is increased with the increase of the magnetic field.

\section{MATHEMATICAL FORMULATION OF THE PROBLEM}

In order to avoid duplication, we make references to our recent paper [2] for the basic equations of motion in a non-rotating two-phase fluid with the same assumptions that are required for the formulation of the problem. The two-phase fluid system is bounded by the two parallel plates at a distance $h$ apart. Thus, the unsteady motion of the two-phase flow in $0 \leq y \leq h$ is governed by the coupled equations with notations similar to those used in [2]:

$$
\begin{aligned}
& \frac{\partial u}{\partial t}=v \frac{\partial^{2} u}{\partial y^{2}}+\frac{k}{\tau}(v-u)-n u \\
& \frac{\partial v}{\partial t}=\frac{1}{\tau}(v-u)
\end{aligned}
$$


where $u(y, t)$ and $\mathrm{v}(y, t)$ represent the fluid and the particle velocities respectively, $k=m N_{0} / \rho$ is the ratio of the mass density of the particles and the fluid density, $\tau$ is the relaxation time of the particles, and $n=\sigma_{0} B_{0}^{2} \rho$ is the hydromagnetic parameter.

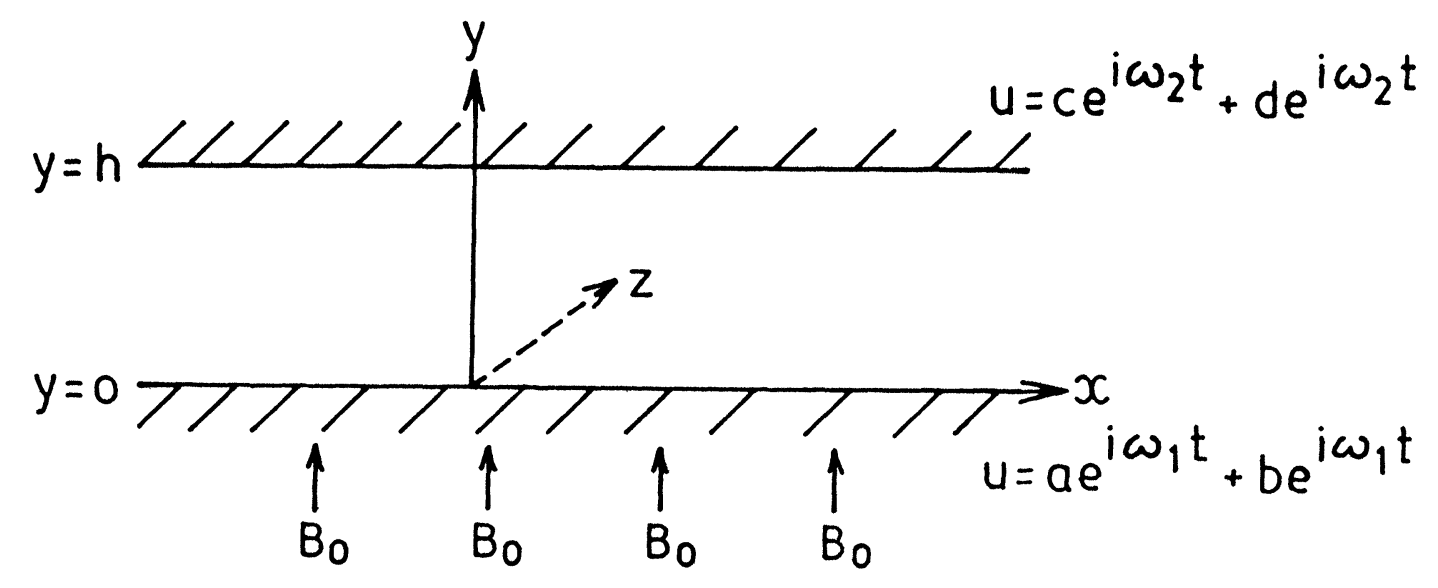

Fig.1 Geometry of the flow

The geometry of the flow configuration is shown in Figure 1. The flow is generated by the non-torsional oscillations of the bounding plates. Consequently, equations (2.1) - (2.2) are to be solved subject to no-slip conditions at the plates:

$$
\begin{gathered}
u=a e^{i \omega_{1} t}+b e^{-i \omega_{1} t} \text { at } y=0, t>0 \\
u=c e^{i \omega_{2} t}+d e^{-i \omega_{2} t} \text { at } y=h, t>0
\end{gathered}
$$

where $a, b, c$, and $d$ are complex constants such that $u$ becomes real on the plates.

The initial conditions of the problem are

$$
u=0, v=0 \text { at } t \leq 0 \text { for all } y \text { in }(0, h) .
$$

It is convenient to introduce the non-dimensional flow variables

$$
y^{\prime}=\frac{y}{h^{\prime}}, t^{\prime}=\frac{v t}{h^{2}}, u^{\prime}=\frac{u h}{v}, v^{\prime}=\frac{v h}{v}
$$

and the non-dimensional flow parameters

$$
\begin{gathered}
T=\frac{\tau v}{h^{2}}, \quad n^{\prime}=\frac{n h^{2}}{v}, \quad\left(\sigma_{1}, \sigma_{2}\right)=\frac{h^{2}}{v}\left(\omega_{1}, \omega_{2}\right), \\
\left(a^{\prime}, b^{\prime}, c^{\prime}, d^{\prime}\right)=\frac{h}{v}(a, b, c, d) .
\end{gathered}
$$

In terms of the dimensionless quantities, equations (2.1) and (2.2) along with the boundary and initial conditions (2.3)-(2.5ab) become, on dropping the primes, 


$$
\begin{gathered}
\frac{\partial u}{\partial t}=\frac{\partial^{2} u}{\partial y^{2}}+\frac{k}{T}(\mathrm{v}-u)-n u, \\
\frac{\partial \mathrm{v}}{\partial t}=\frac{1}{T}(u-\mathrm{v}), \\
u=a e^{i \sigma_{1} t}+b e^{-i \sigma_{1} t} \text { on } y=0, t>0, \\
u=c e^{i \sigma_{2} t}+d e^{-i \sigma_{2} t} \text { on } y=1, t>0, \\
u=v=0 \text { at } t \leq 0 \text { for all } y .
\end{gathered}
$$

In order to solve the initial value problem, we introduce the Laplace transform $\bar{u}(y, s)$ of $u(y, t)$ defined in Myint-U and Debnath [3].

Applying the Laplace transform in (2.8) - (2.11) with initial conditions, we obtain the transformed solution with $s$ as the Laplace transform variable

$$
\bar{u}(y, s)=\left(\frac{a}{s-i \sigma_{1}}+\frac{b}{s+i \sigma_{1}}\right) \frac{\sinh p(1-y)}{\sinh p}+\left(\frac{c}{s-i \sigma_{2}}+\frac{d}{s+i \sigma_{2}}\right) \frac{\sinh p y}{\sinh p}
$$

where

$$
p^{2}=\frac{s k}{1+s T}+s+n
$$

We next apply inverse Laplace transformation to obtain the exact solution for the fluid velocity field in the form

$$
\begin{aligned}
u(y, t)= & a\left[\left(\mathrm{~A}_{1} \cos \sigma_{1} t-B_{1} \sin \sigma_{1} t\right)+i\left(B_{1} \cos \sigma_{1} t+A_{1} \sin \sigma_{1} t\right)\right] \\
& +b\left[\left(\mathrm{~A}_{1} \cos \sigma_{1} t-B_{1} \sin \sigma_{1} t\right)-i\left(B_{1} \cos \sigma_{1} t+A_{1} \sin \sigma_{1} t\right)\right] \\
& +c\left[\left(\mathrm{~A}_{2} \cos \sigma_{2} t-B_{2} \sin \sigma_{2} t\right)+i\left(B_{2} \cos \sigma_{2} t+A_{2} \sin \sigma_{2} t\right)\right] \\
& +d\left[\left(\mathrm{~A}_{2} \cos \sigma_{2} t-B_{2} \sin \sigma_{2} t\right)-i\left(B_{1} \cos \sigma_{2} t+A_{2} \sin \sigma_{2} t\right)\right] \\
& -2 \pi \sum_{m=1}^{\infty} m(-1)^{m} \sin m \pi(1-y)\left[\Delta_{1} e^{\lambda_{1} t}+\Delta_{2} e^{\lambda_{2} t}\right] \\
& -2 \pi \sum_{m=1}^{\infty} m(-1)^{m} \sin m \pi y\left[\Delta_{3} e^{\lambda_{1} t}+\Delta_{4} e^{\lambda_{2} t}\right]
\end{aligned}
$$

where

$$
\begin{aligned}
& \text { (2.16) } A_{1}=\frac{1}{k_{3}}\left[k_{1} \cos \mu_{1}(1-y) \sinh \mu_{2}(1-y)+k_{2} \sin \mu_{1}(1-y) \cosh \mu_{2}(1-y)\right] \\
& (2.17) B_{1}=\frac{1}{k_{3}}\left[k_{1} \sin \mu_{1}(1-y) \cosh \mu_{2}(1-y)-k_{2} \cos \mu_{1}(1-y) \sinh \mu_{2}(1-y)\right] \\
& \text { (2.20abc) } \quad k_{1}=\cos \mu_{1} \sinh \mu_{2}, k_{2}=\sin \mu_{1} \cosh \mu_{2}, k_{3}^{2}=k_{1}^{2}+k_{2}^{2} \\
& (2.21 \mathrm{abc}) \quad k_{1}^{\prime}=\cos \mu_{3} \sinh \mu_{4}, k_{2}^{\prime}=\sin \mu_{3} \cosh \mu_{4}, k_{3}^{\prime}=k_{1}^{\prime 2}+k_{2}^{\prime 2}
\end{aligned}
$$




$$
\begin{aligned}
A & =n+\frac{\sigma_{1}^{2} T k}{1+T^{2} \sigma_{1}^{2}}, B=\sigma_{1}\left(1+\frac{k}{1+T^{2} \sigma_{1}^{2}}\right) \\
G & =n+\frac{\sigma_{2}^{2} T k}{1+T \sigma_{2}^{2}}, H=\sigma_{2}\left(1+\frac{k}{1+T^{2} \sigma_{2}^{2}}\right) \\
\left(\mu_{1}, \mu_{2}\right)= & \left(\frac{\sqrt{A^{2}+B^{2}} \mp A}{2}\right)^{\frac{1}{2}},\left(\mu_{3}, \mu_{4}\right)=\left(\frac{\sqrt{G^{2}+H^{2}} \mp G}{2}\right)^{\frac{1}{2}} \\
\Delta_{r}= & {\left[\frac{a}{\lambda_{r}-i \sigma_{1}}+\frac{b}{\lambda_{r}+i \sigma_{1}}\right]\left[1+\frac{k}{\left(1+\lambda_{r} T\right)^{2}}\right]^{-1}, \mathrm{r}=1,2 } \\
\Delta_{3} & =\left[\frac{c}{\lambda_{1}-i \sigma_{2}}+\frac{d}{\lambda_{1}+i \sigma_{2}}\right]\left[1+\frac{k}{\left(1+\lambda_{1} T\right)^{2}}\right]^{-1} \\
\Delta_{4} & =\left[\frac{c}{\lambda_{2}-i \sigma_{2}}+\frac{d}{\lambda_{2}+i \sigma_{2}}\right]\left[1+\frac{k}{\left(1+\lambda_{2} T\right)^{2}}\right]^{-1} \\
& \left(2 T \lambda_{1}, 2 T \lambda_{2}\right)=-\left[1+k+T n+T m^{2} \pi^{2}\right] \\
& \pm\left[\left\{1+k+T n+T m^{2} \pi^{2}\right\}^{2}-4 T\left(n+m^{2} \pi^{2}\right)\right]^{1 / 2} .
\end{aligned}
$$

Application of the convolution theorem for the Laplace transform to (2.9) gives the exact solution for the particle velocity.

$$
\begin{aligned}
\mathrm{v}(y, t)= & \frac{a}{1+T^{2} \sigma_{1}^{2}}\left[\left\{\left(A_{1}+B_{1} T \sigma_{1}\right)\left(\cos \sigma_{1} t-e^{-t / T}\right)-\left(B_{1}-A_{1} T \sigma_{1}\right) \sin \sigma_{1} t\right\}\right. \\
& \left.+i\left\{\left(B_{1}-A_{1} T \sigma_{1}\right)\left(\cos \sigma_{1} t-e^{-t / T}\right)+\left(A_{1}+B_{1} T \sigma_{1}\right) \sin \sigma_{1} t\right\}\right] \\
& +\frac{b}{1+T^{2} \sigma_{1}^{2}}\left[\left\{\left(A_{1}+B_{1} T \sigma_{1}\right)\left(\cos \sigma_{1} t-e^{-t / T}\right)-\left(B_{1}-A_{1} T \sigma_{1}\right) \sin \sigma_{1} t\right\}\right. \\
& \left.-i\left\{\left(B_{1}-A_{1} T \sigma_{1}\right)\left(\cos \sigma_{1} t-e^{-t / T}\right)+\left(A_{1}+B_{1} T \sigma_{1}\right) \sin \sigma_{1} t\right\}\right] \\
& +\frac{c}{1+T^{2} \sigma_{2}^{2}}\left[\left\{\left(A_{2}+B_{2} T \sigma_{2}\right)\left(\cos \sigma_{2} t-e^{-t / T}\right)-\left(B_{2}-A_{2} T \sigma_{2}\right) \sin \sigma_{2} t\right\}\right. \\
& \left.+i\left\{\left(B_{2}-A_{2} T \sigma_{2}\right)\left(\cos \sigma_{2} t-e^{-t / T}\right)+\left(A_{2}+B_{2} T \sigma_{2}\right) \sin \sigma_{2} t\right\}\right] \\
& +\frac{d^{2}}{1+T^{2} \sigma_{2}^{2}}\left[\left\{\left(A_{2}+B_{2} T \sigma_{2}\right)\left(\cos \sigma_{2} t-e^{-t / T}\right)-\left(B_{2}-A_{2} T \sigma_{2}\right) \sin \sigma_{2} t\right\}\right.
\end{aligned}
$$


(2.9), cont. $\left.-i\left\{\left(B_{2}-A_{2} T \sigma_{2}\right)\left(\cos \sigma_{2} t-e^{-t / T}\right)+\left(A_{2}+B_{2} T \sigma_{2}\right) \sin \sigma_{2} t\right\}\right]$

$$
\begin{aligned}
& -2 \pi \sum_{m=1}^{\infty} m(-1)^{m} \sin m \pi(1-y)\left[\frac{e^{\lambda_{1} t}-e^{-t / T}}{\lambda_{1}+\frac{1}{T}} \Delta_{1}+\frac{e^{\lambda_{2} t}-e^{-t / T}}{\lambda_{2}+\frac{1}{T}} \Delta_{2}\right] \\
& -2 \pi \sum_{m=1}^{\infty} m(-1)^{m} \sin m \pi y\left|\frac{e^{\lambda_{1} t}-e^{-t / T}}{\lambda_{1}+\frac{1}{T}} \Delta_{3}+\frac{e^{\lambda_{2} t}-e^{-t / T}}{\lambda_{2}+\frac{1}{T}} \Delta_{4}\right| .
\end{aligned}
$$

The results (2.15) and (2.29) describe respectively the fluid and the particle velocities for the general case. Many particular results follow easily from these solutions. For example, if $a=b=0, c=d=V / 2$ and $\omega_{2}=\omega$ then (2.15) and (2.29)reduce to those given by Mitra and Bhattacharyya [4]. However, if $\omega_{1}=0$ and $\omega_{2}=0$, the results corresponding to Mitra and Bhattacharyya [5] will emerge. Moreover, when $(a, b)=\left(u_{0} / 2 i,-u_{0} / 2 i\right)$ and $(c, d)=\left(u_{1} / 2 i,-u_{1} / 2 i\right)$ are substituted in (2.15) and (2.29) with $\sigma_{2}=\sigma_{1}=\sigma$, the resulting solutions provide an extension of the work of Stanisc et al. [6] for the two-phase fluid particle system.

\section{THE WALL SHEAR STRESSES}

The shear stress, $S_{0}$, at the lower plate exerted by the conducting dusty fluid is given by

$$
\begin{aligned}
S_{0}=-\left(\frac{\partial u}{\partial y}\right)_{y=0}= & a\left[\left(L_{1} \cos \sigma_{1} t-M_{1} \sin \sigma_{1} t\right)+i\left(M_{1} \cos \sigma_{1} t+L_{1} \sin \sigma_{1} t\right)\right] \\
& +b\left[\left(L_{1} \cos \sigma_{1} t-M_{1} \sin \sigma_{1} t\right)-i\left(M_{1} \cos \sigma_{1} t+L_{1} \sin \sigma_{1} t\right)\right] \\
& +c\left[\left(M_{2} \sin \sigma_{2} t-L_{2} \cos \sigma_{2} t\right)-i\left(M_{2} \cos \sigma_{2} t+L_{2} \sin \sigma_{2} t\right)\right] \\
& +d\left[\left(M_{2} \sin \sigma_{2} t-L_{2} \cos \sigma_{2} t\right)+i\left(M_{2} \cos \sigma_{2} t+L_{2} \sin \sigma_{2} t\right)\right] \\
& -2 \pi^{2} \sum_{m=1}^{\infty} m^{2}\left[e^{\lambda_{1} t} \Delta_{1} e^{\lambda_{2^{t}}} \Delta_{2}\right] \\
& +2 \pi^{2} \sum_{m=1}^{\infty}(-1)^{m} m^{2}\left[e^{\lambda_{1} t} \Delta_{3+} e^{\lambda_{2} t} \Delta_{4}\right]
\end{aligned}
$$

where

$$
\begin{aligned}
& L_{1}=\frac{1}{k_{3}}\left[\mu_{1} \cos \mu_{1} \sin \mu_{1}+\mu_{2} \cosh \mu_{2} \sinh \mu_{2}\right] \\
& M_{1}=\frac{1}{k_{3}}\left[\mu_{1} \sinh \mu_{2} \cosh \mu_{2}-\mu_{2} \cos \mu_{1} \sin \mu_{1}\right] \\
& L_{2}=\frac{1}{k_{3}^{\prime}}\left(\mu_{4} k_{1}^{\prime}+\mu_{3} k_{2}^{\prime}\right) \text { and } M_{2}=\frac{1}{k_{3}^{\prime}}\left(\mu_{3} k_{1}^{\prime}-\mu_{4} k_{2}^{\prime}\right) .
\end{aligned}
$$


Similarly, using (2.15), the shear stress, $S_{1}$, at the upper oscillating plate in dimensionless form is expressed as

$$
\begin{aligned}
S_{1}=-\left(\frac{\partial u}{\partial y}\right)_{y=1}= & a\left[\left(L_{1}^{\prime} \cos \sigma_{1} t+M_{1}^{\prime} \sin \sigma_{1} t\right)-i\left(M_{1}^{\prime} \cos \sigma_{1} t-L_{1}^{\prime} \sin \sigma_{1} t\right)\right] \\
& +b\left[\left(L_{1}^{\prime} \cos \sigma_{1} t+M_{1}^{\prime} \sin \sigma_{1} t\right)+i\left(M_{1}^{\prime} \cos \sigma_{1} t-L_{1}^{\prime} \sin \sigma_{1} t\right)\right] \\
& +c\left[\left(M_{2}^{\prime} \sin \sigma_{2} t+L_{2}^{\prime} \cos \sigma_{2} t\right)-i\left(M_{2}^{\prime} \cos \sigma_{2} t-L_{2}^{\prime} \sin \sigma_{2} t\right)\right] \\
& +d\left[\left(M_{2}^{\prime} \sin \sigma_{2} t+L_{2}^{\prime} \cos \sigma_{2} t\right)+i\left(M_{2}^{\prime} \cos \sigma_{2} t+L_{2}^{\prime} \sin \sigma_{2} t\right)\right] \\
& -2 \pi^{2} \sum_{m=1}^{\infty} m^{2}(-1)^{m}\left[\Delta_{1} e^{\lambda_{1} t}+\Delta_{2} e^{\lambda_{2} t}\right] \\
& +2 \pi^{2} \sum_{m=1}^{\infty} m^{2}\left[\Delta_{3} e^{\lambda_{1} t}+\Delta_{4} e^{\lambda_{2} t}\right],
\end{aligned}
$$

where

$$
\begin{gathered}
L_{1}^{\prime}=\frac{1}{k_{3}^{\prime}}\left(k_{1} \mu_{2}+k_{2} \mu_{1}\right) \text { and } M_{1}^{\prime}=\frac{1}{k_{3}^{\prime}}\left(k_{2} \mu_{2}-k_{1} \mu_{1}\right) \\
L_{2}^{\prime}=\frac{1}{k_{3}^{\prime}}\left[\mu_{3} \sinh \mu_{3} \cosh \mu_{3}+\mu_{4} \sinh \mu_{4} \cosh \mu_{4}\right] \\
M_{2}^{\prime}=\frac{1}{k_{3}^{\prime}}\left[\mu_{3} \cosh \mu_{4} \sinh \mu_{4}+\mu_{4} \cosh \mu_{3} \sinh \mu_{3}\right] .
\end{gathered}
$$

The volume of the conducting dusty fluid discharged per unit breadth between the plates is

$$
Q=\int_{0}^{1} u d y .
$$

Using (2.15) we obtain

where

$$
\begin{aligned}
Q= & a\left[\left(N_{1} \cos \sigma_{1} t-N_{2} \sin \sigma_{1} t\right)+i\left(N_{2} \cos \sigma_{1} t+N_{1} \sin \sigma_{1} t\right)\right] \\
& +b\left[\left(N_{1} \cos \sigma_{1} t-N_{2} \sin \sigma_{1} t\right)-i\left(N_{2} \cos \sigma_{1} t+N_{1} \sin \sigma_{1} t\right)\right] \\
& +c\left[\left(N_{3} \cos \sigma_{2} t-N_{4} \sin \sigma_{2} t\right)+i\left(N_{4} \cos \sigma_{2} t+N_{3} \sin \sigma_{2} t\right)\right] \\
& +d\left[\left(N_{3} \cos \sigma_{2} t-N_{4} \sin \sigma_{2} t\right)-i\left(N_{4} \cos \sigma_{2} t+N_{3} \sin \sigma_{2} t\right)\right] \\
& -2 \sum_{m=1}^{\infty}\left[(-1)^{m}-1\right]\left[\Delta_{1} e^{\lambda_{1} t}+\Delta_{2} e^{\lambda_{2} t}\right]-2 \sum_{m=1}^{\infty}\left[(-1)^{m}-1\right]\left[\Delta_{3} e^{\lambda_{1} t}+\Delta_{4} e^{\lambda_{2} t}\right]
\end{aligned}
$$

$$
\begin{aligned}
& N_{1}=\left[\left(\mu_{2} k_{1}-k_{2} \mu_{1}\right)\left(\cosh \mu_{2} \cos \mu_{1}-1\right)+\left(k_{1} \mu_{1}+k_{2} \mu_{2}\right) \sin \mu_{1} \sinh \mu_{2}\right] / k_{3}\left(\mu_{1}^{2}+\mu_{2}^{2}\right) \\
& N_{2}=\left[\left(k_{1} \mu_{1}-k_{2} \mu_{2}\right)\left(1-\cosh \mu_{2} \cos \mu_{1}\right)+\left(k_{1} \mu_{2}-k_{2} \mu_{1}\right) \sin \mu_{1} \sinh \mu_{2}\right] / k_{3}\left(\mu_{1}^{2}+\mu_{2}^{2}\right) \\
& N_{3}=\left[\left(k_{1}^{\prime} \mu_{3}+k_{2}^{\prime} \mu_{4}\right) \sinh \mu_{4} \sin \mu_{3}+\left(k_{1}^{\prime} \mu_{4}+k_{2}^{\prime} \mu_{3}\right)\left(\cosh \mu_{4} \cos \mu_{3}-1\right)\right] / k_{3}^{\prime}\left(\mu_{3}^{2}+\mu_{4}^{2}\right) \\
& N_{4}=\left[\left(k_{1}^{\prime} \mu_{3}+k_{2}^{\prime} \mu_{4}\right)\left(1-\cosh \mu_{4} \cos \mu_{3}\right)+\left(k_{1}^{\prime} \mu_{4}+k_{2}^{\prime} \mu_{3}\right) \sin \mu_{3} \sinh \mu_{4} 1 / k_{3}^{\prime}\left(\mu_{3}^{2}+\mu_{4}^{2}\right) .\right.
\end{aligned}
$$




\section{NUMERICAL RESULTS WITH GRAPHICAL REPRESENTATIONS}

To proceed with the numerical evaluation of the general results (2.15), (2.29), and (3.1) in some particular cases, we first suppose that the time period of one complete oscillation of the lower plate is $T_{1}$ while that of the upper plate is $T_{2}$. Consequently, $\sigma_{1}=2 \pi / T_{1}$ and $\sigma_{2}=2 \pi / T_{2}$ with $\sigma=T_{1} / T_{2}$. We now discuss the relevant results corresponding to three different types of movement of the plates: (i) $\sigma<1$, (ii) $\sigma=1$, and (iii) $\sigma>1$. In order that the plates oscillate in phase with each other in all the three cases we put $a=b=1 / 2$ and $c=d=1 / 2$ in the general results. The modified form of the general results for the fluid, the particle velocities and the wall shear stress on the lower plate are then evaluated numerically for various values of the flow parameters $k, T, n, T_{1}$, and $\sigma$ and also for different $y$ and $t$. The velocity profiles are represented graphically for values of $y$ from 0 to 1 when $t=T_{1} / 8,2 T_{1} / 8,3 T_{1} / 8,4 T_{1} / 8,5 T_{1} / 8,6 T_{1} / 8,7 T_{1} / 8$, and $T_{1}$.

In figures 2,3 , and 4 , the velocity profiles for the conducting dusty fluid are shown for different values of $k$, the concentration of the dust particles, when $\sigma=0.5,1.0$, and 1.5 respectively, while the other parameters $T, n$, and $T_{1}$ are kept to the values $0.1,0.5$, and 1.0 in each case. The variation of these velocity profiles due to increasing values of $T_{1}$, i.e. when $T_{1}=10.0$, is presented in figures 5,6, and 7. An increase in the concentration of the particles retards the fluid motion when the time period of the oscillation of the plates is small, i.e., their frequencies are high. On the other hand, when the time periods of

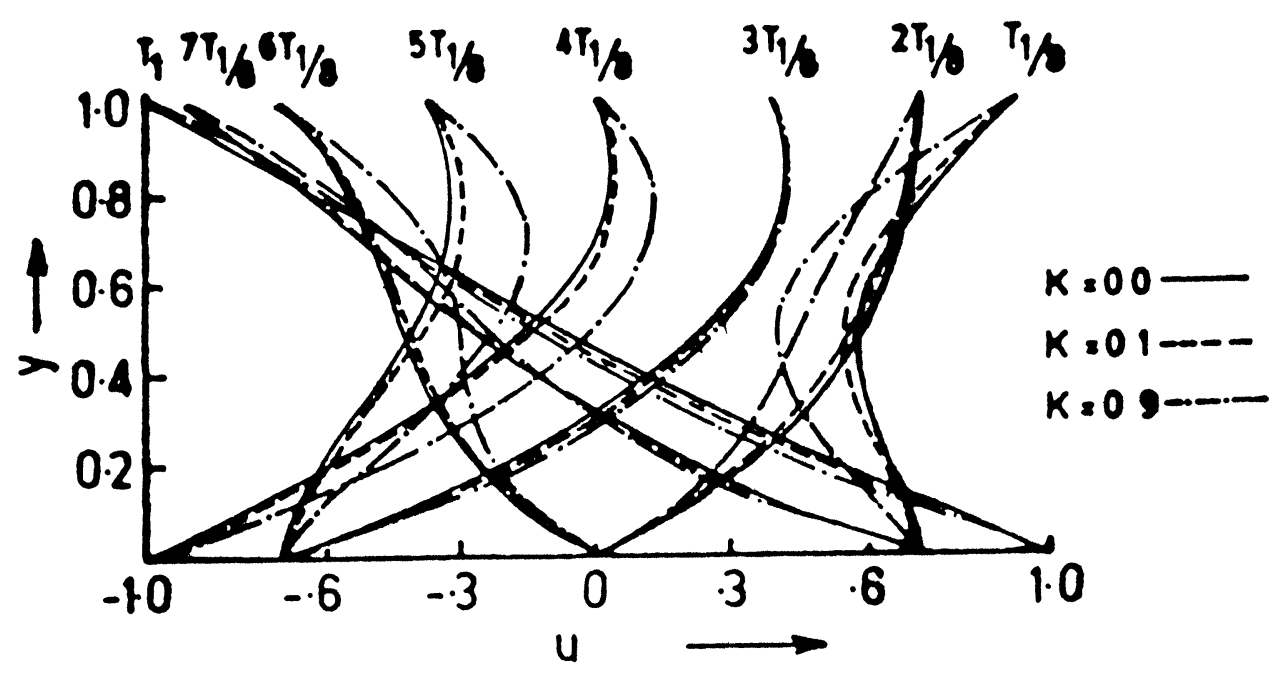

Fig.2. Velocity distribution for conducting dusty fluid for different values of $K$ when $T=0.1 \quad N=0.5 \quad T_{1}=1.0$ and $\sigma=0.5\left(T_{1}<T_{2}\right)$ 


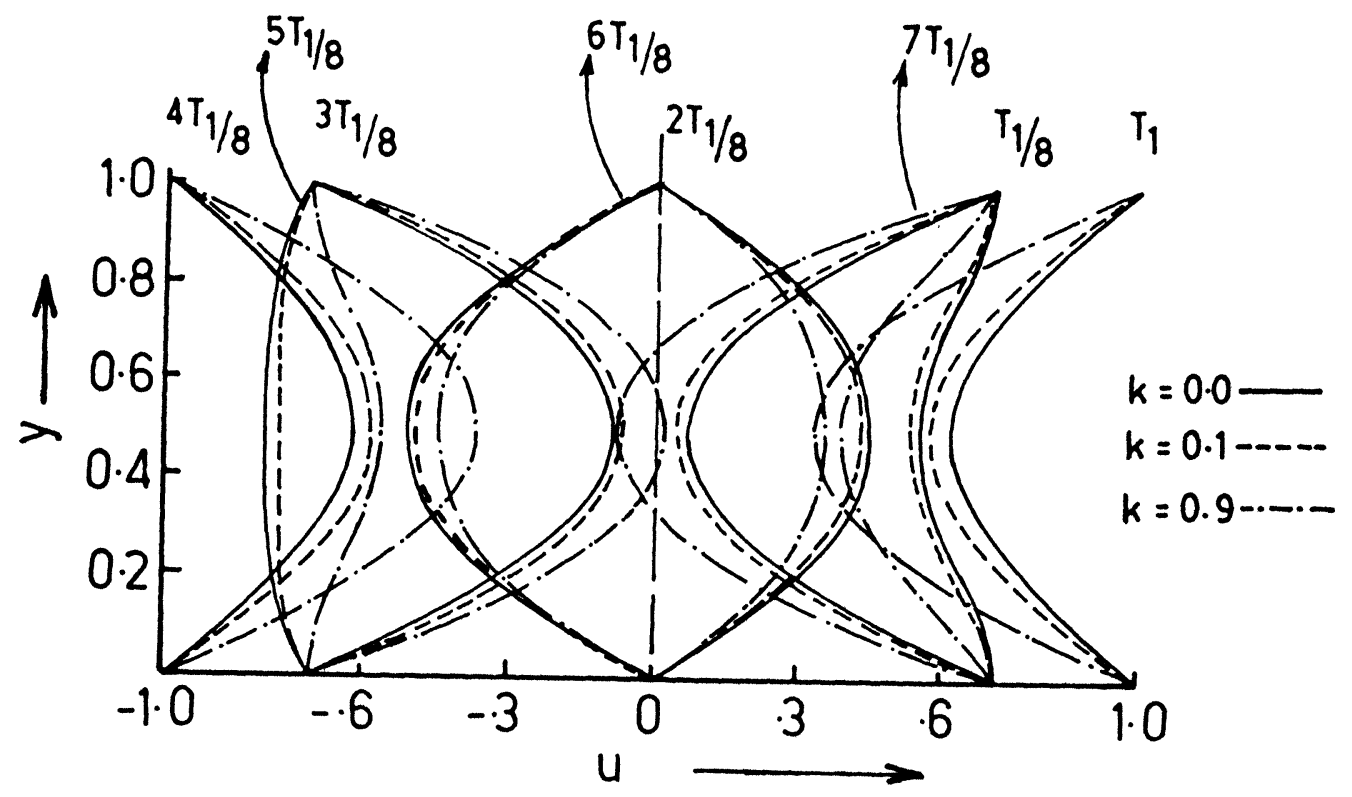

Fig.3. Velocity distribution for conducting dusty fluid for different values of $k$ when $T=0.1, n=0.5, T_{1}=1.0$ and $\sigma=1.0 \quad\left(T_{1}=T_{2}\right)$

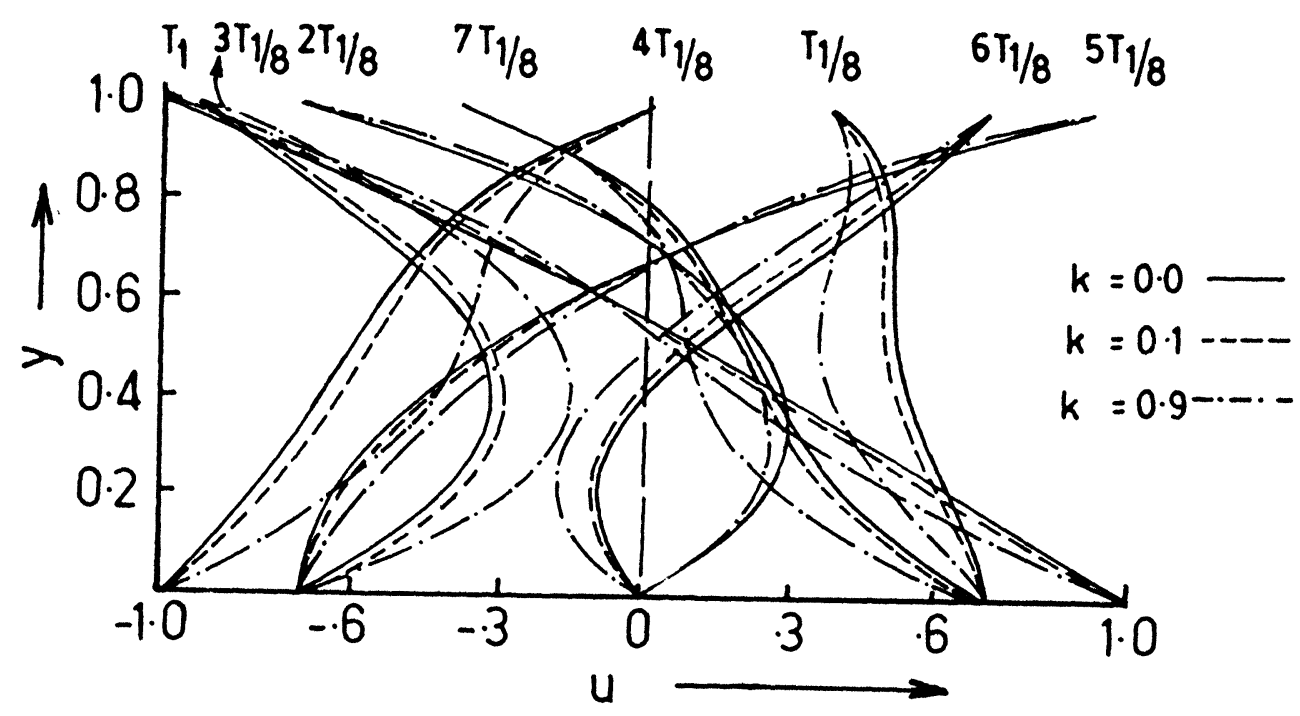

Fig. 4. Velocity distrbution for conducting dusty fluid for different values of $k$ when $T=0.1, n=0.5, T_{1}=1.0$ and $\sigma=1.5\left(T_{1}>T_{2}\right)$

oscillation of the plates are large, i.e., their frequencies of oscillation are low, the fluid motion is accelerated by the presence of the particles at the beginning and persists with this effect until the equilibrium is reached when the particles exert their influence to resist the 
fluid motion (see fig. 5, 6, 7). We further notice from the figures mentioned above that the effect of particles is more pronounced near the plate having a time period larger than the other provided the time periods of oscillation of both the plates are small and different.

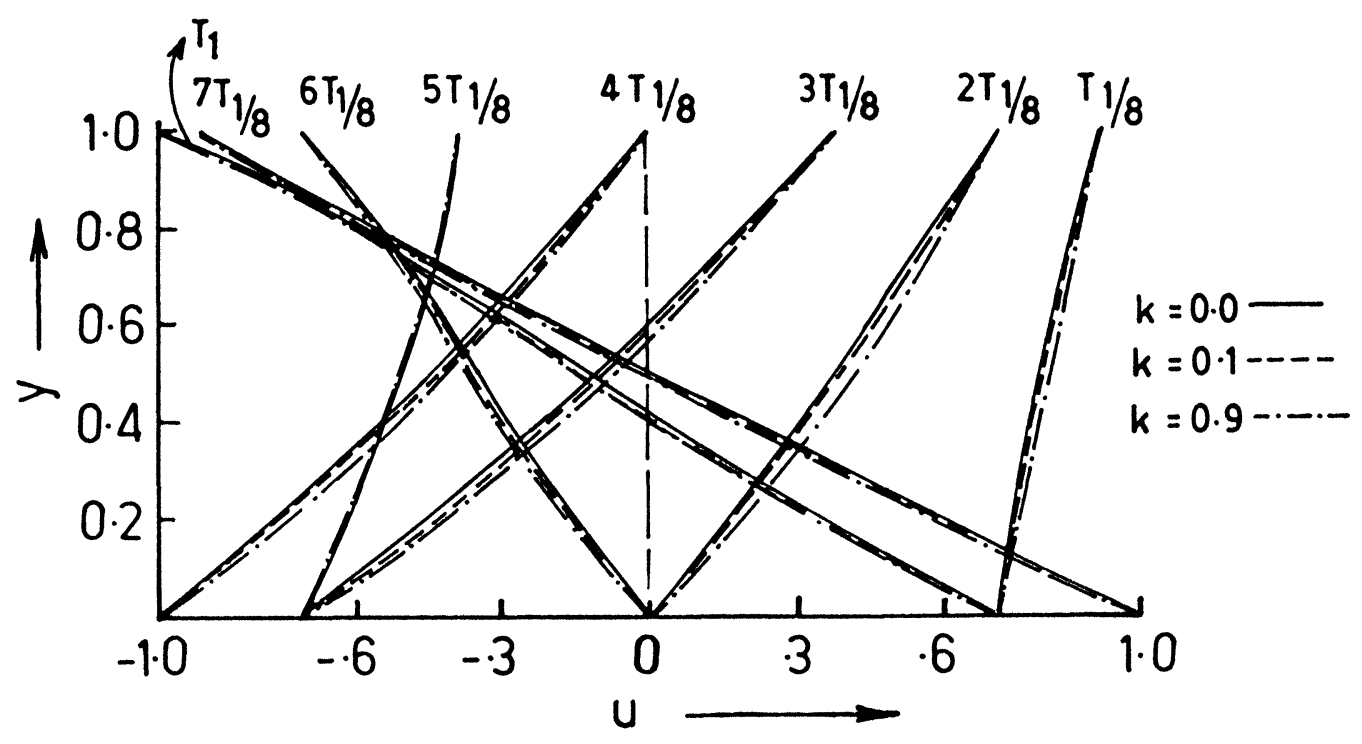

Fig.5. Velocity distribution for conducting dusty fluid for different values of $k$ when $T=0.1, n=0.5, T_{1}=10.0$ and $\sigma=0.5\left(T_{1}<T_{2}\right)$

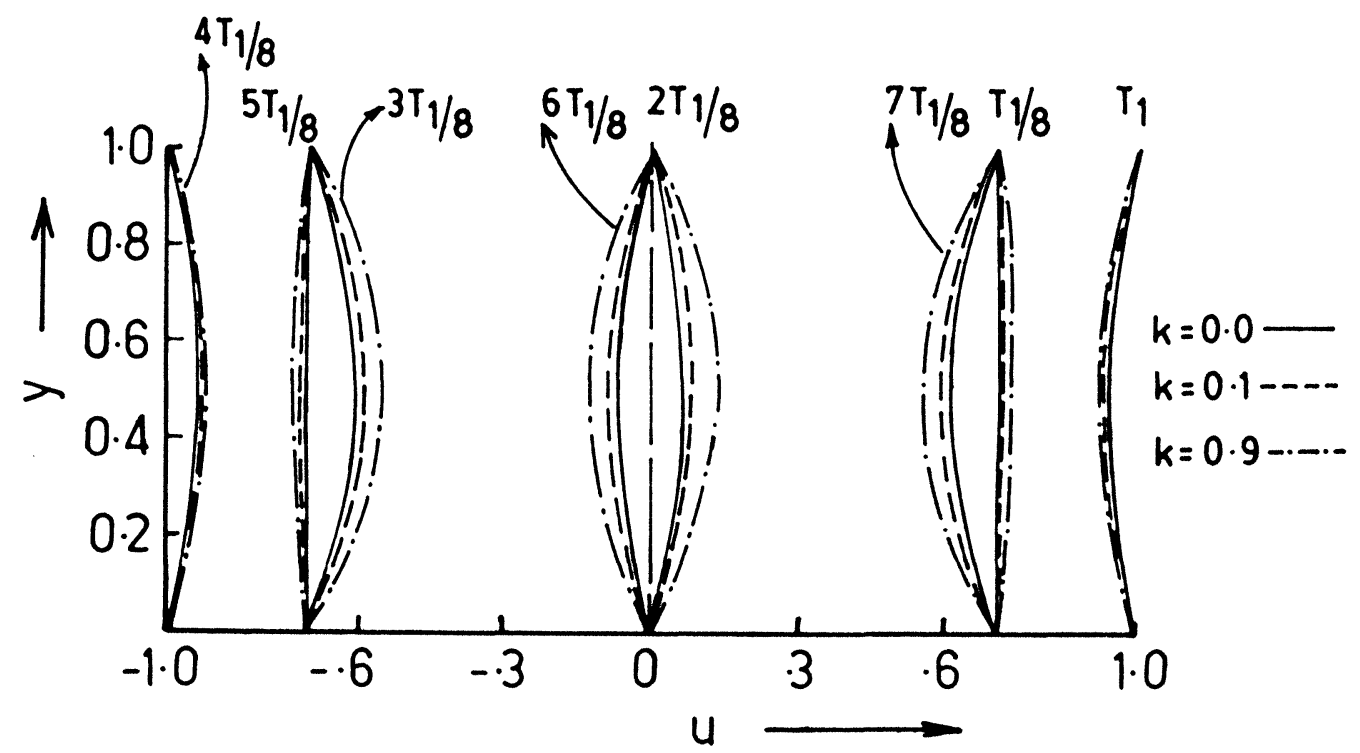

Fig.6. Velocity distribution for conducting dusty fluid for different values of $k$ when $T=0.1, n=0.5, T_{1}=10.0$ and $\sigma=1.0\left(T_{1}=T_{2}\right)$ 
In figures 8 to 13 the velocity profiles for the conducting dusty fluid are shown for different values of the magnetic field in each of the three different cases of $\sigma$ when $T_{1}$ is 1.0 and 10.0 respectively. It is clear from the figures that the magnetic field has a strong damping effect on the fluid velocity irrespective of the values of $\sigma$ and $T_{1}$.

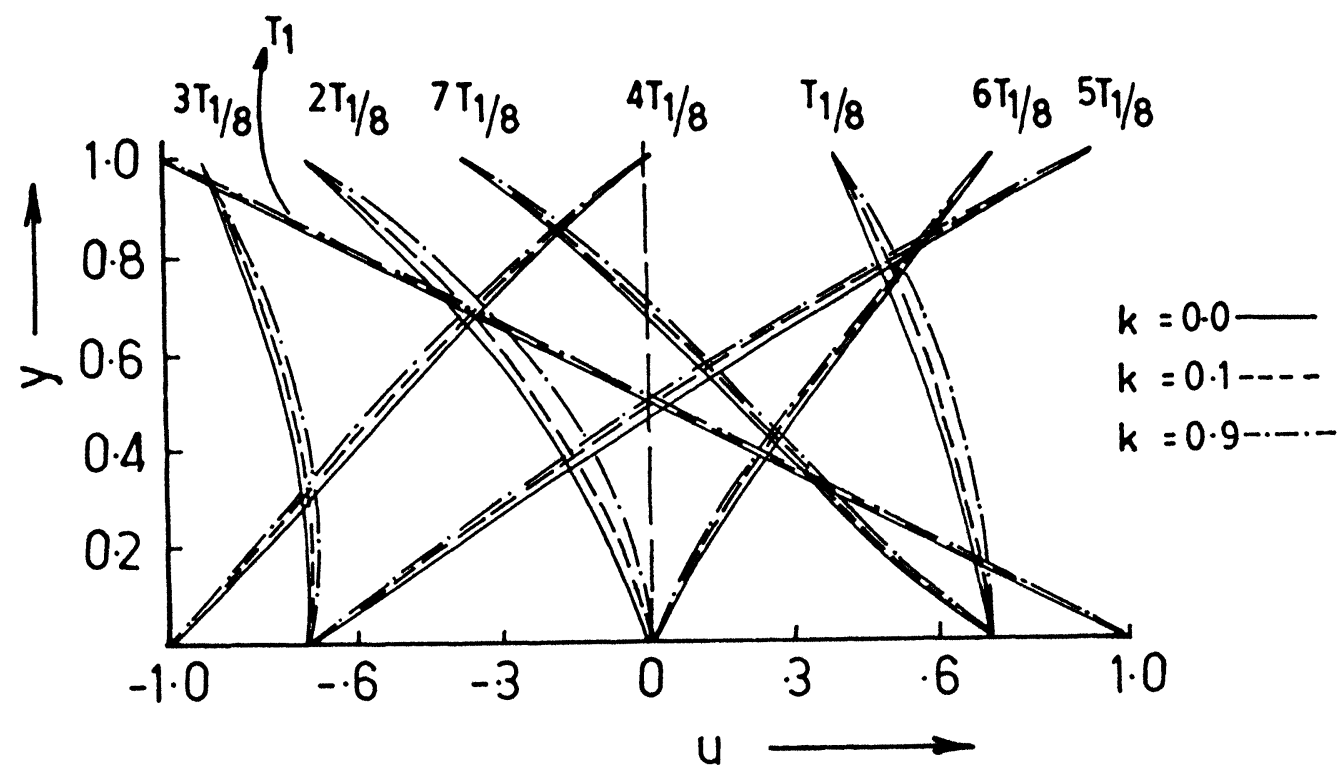

Fig.7. Velocity distributionfor conducting dusty fluid for different values of $k$ when $T=0.1, n=0.5, T_{1}=10.0$ and $\sigma=1.5\left(T_{1}>T_{2}\right)$

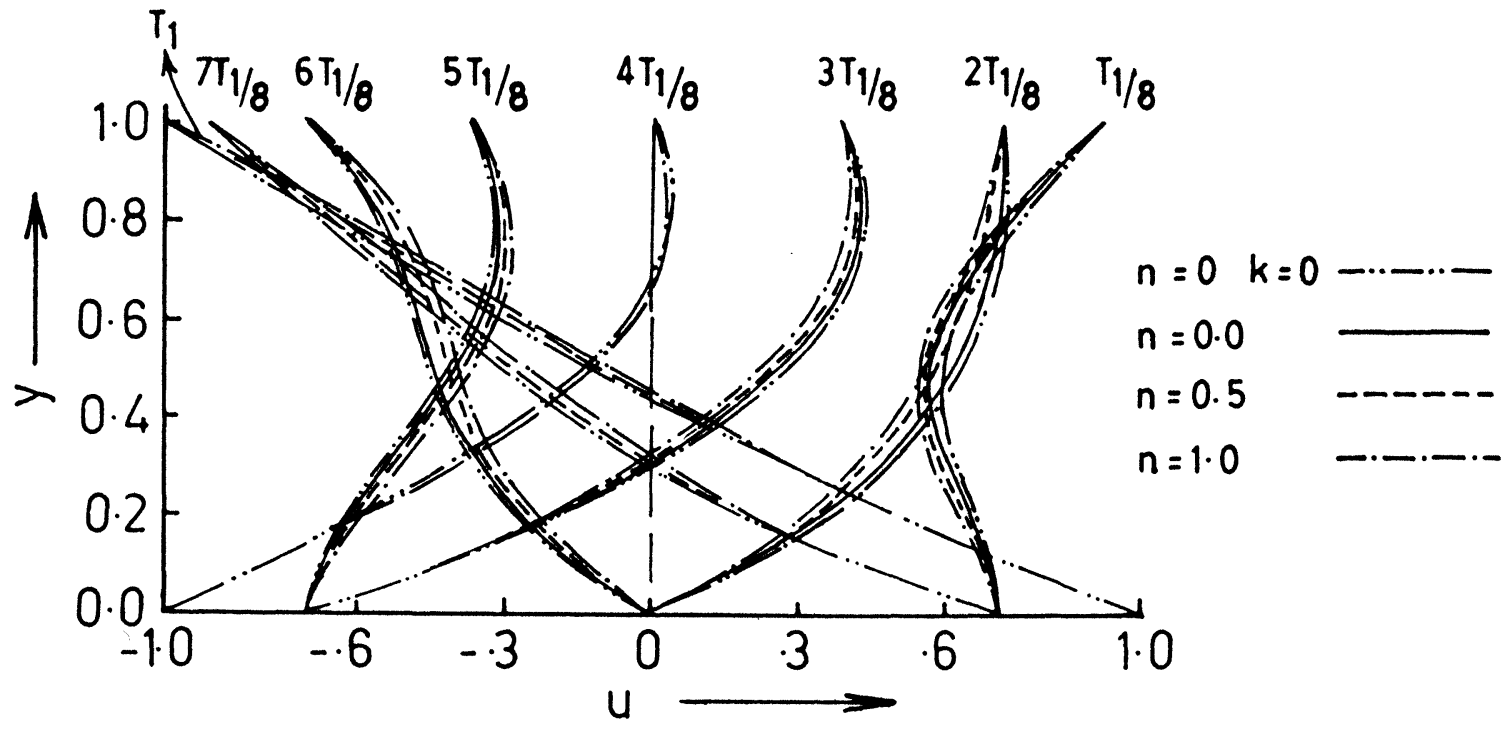

Fig. 8. Velocity distribution for conducting dusty fluid for different values of $n$ when $k=0.1, T=0.3, T_{1}=1.0$ and $\sigma=0.5$ $\left(T_{1}<T_{2}\right)$ 


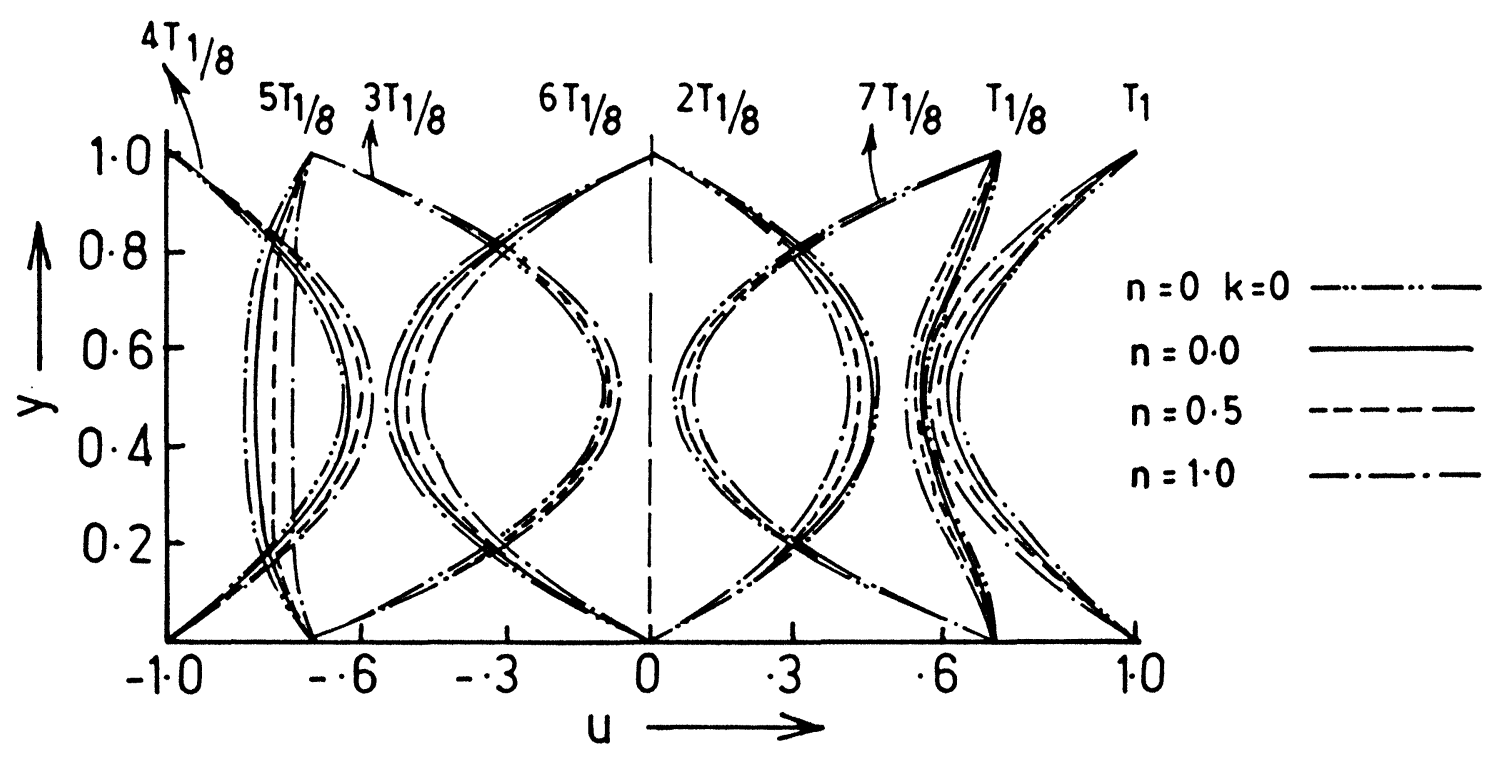

Fig.9. Velocity distribution for conducting dusty fluid for different values of $n$ when $k=0.1, T=0 \cdot 3, T_{1}=1 \cdot 0$ and $\sigma=1$ $\left(T_{1}=T_{2}\right)$

The effect of the magnetic field on the particle velocity is shown in figures 14,15 , and 16 from which one can easily conclude that the speed of the particles decreases with the increase of the magnetic field.

Finally, figures 17, 18, and 19 represent the oscillation of the drag on the lower plate corresponding to three different values of $\sigma$ when the time period of the oscillation of the lower plate is 10.0. It is seen that the drag oscillation is larger when the plates oscillate with different time periods than when they oscillate with the same time period. After a time $\mathrm{t} \approx 1.2$ (i.e. $t \approx 4 \tau$ in dimensional form) the magnitude of the drag is found to increase with the increasing particle concentration for all values of the magnetic parameter $n$ when $\sigma=0.5$ and 1.0. The reverse effect is also seen when $\sigma=1.5$. In this case the drag diminishes with the increase of particle concentration for all $n$. The non-equilibrium stress values are generally considered up to the time limit $t \approx \tau$ in the case number of a single plate configuration which is $t \approx 4 \tau$ in our case. 


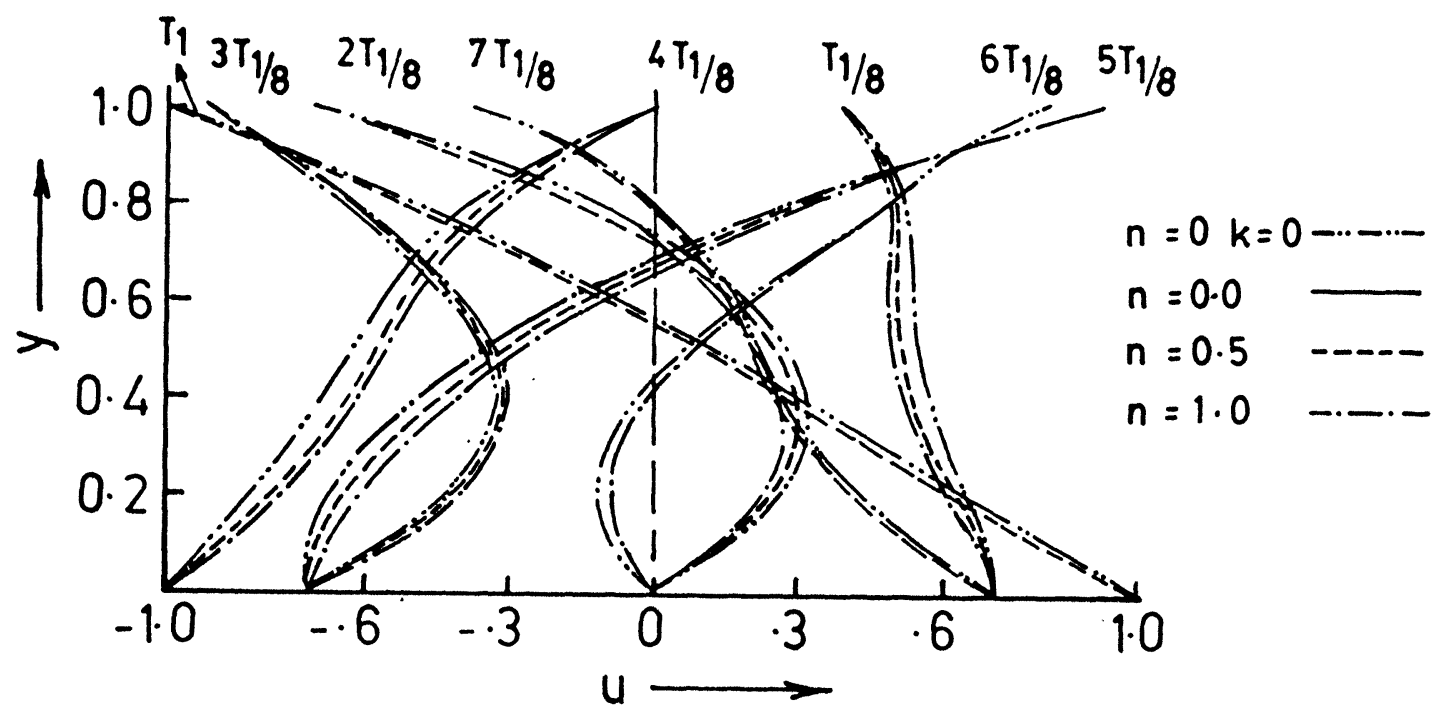

Fig.10 Velocity distribution for conducting dusty fluid for different values of $n$ when $k=0.1, T=0.3, T_{1}=1.0$ and $\sigma=1.5$ $\left(T_{1}>T_{2}\right)$

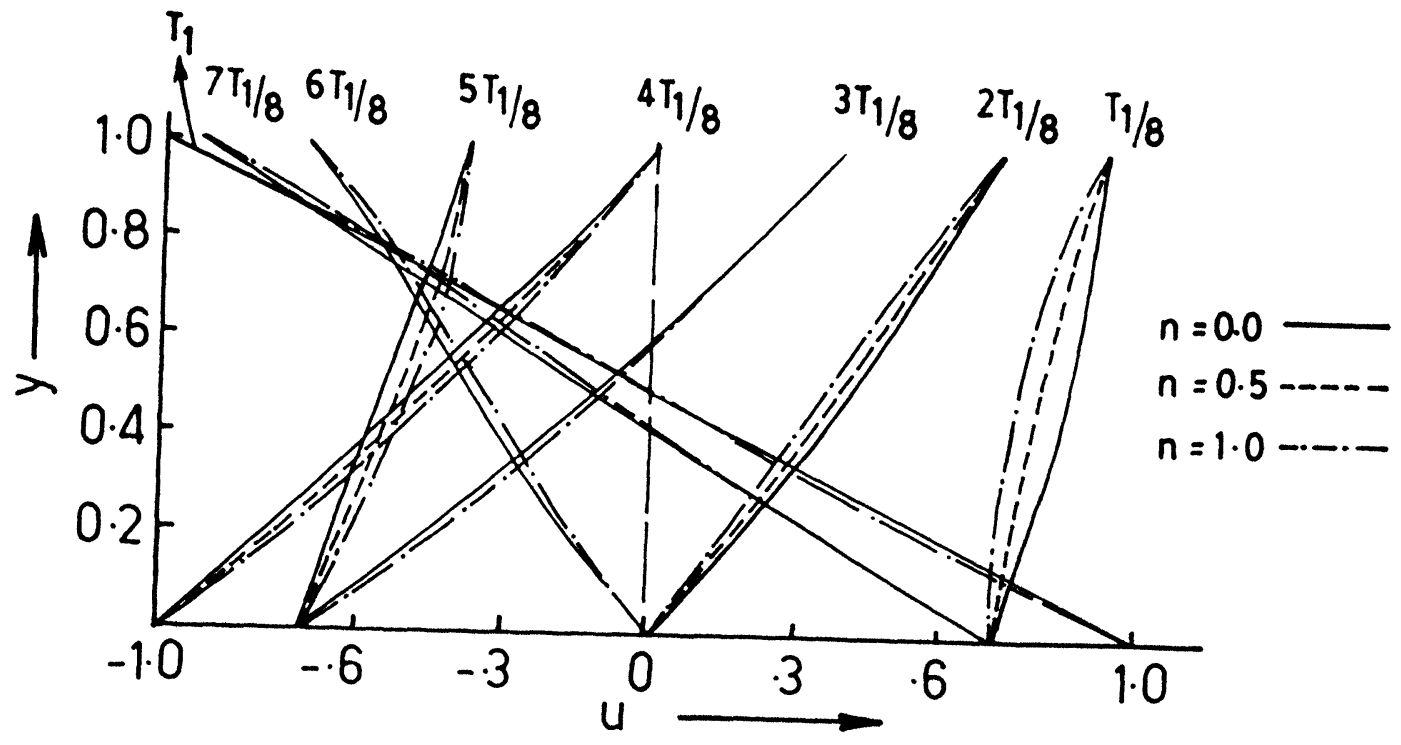

Fig.11. Velocity distribution for conducting dusty fluid for different values of $n$ when $k=0.1 T=0.1 \quad T_{1}=10.0$ and $O=0.5$ $\left(T_{1}<T_{2}\right)$ 


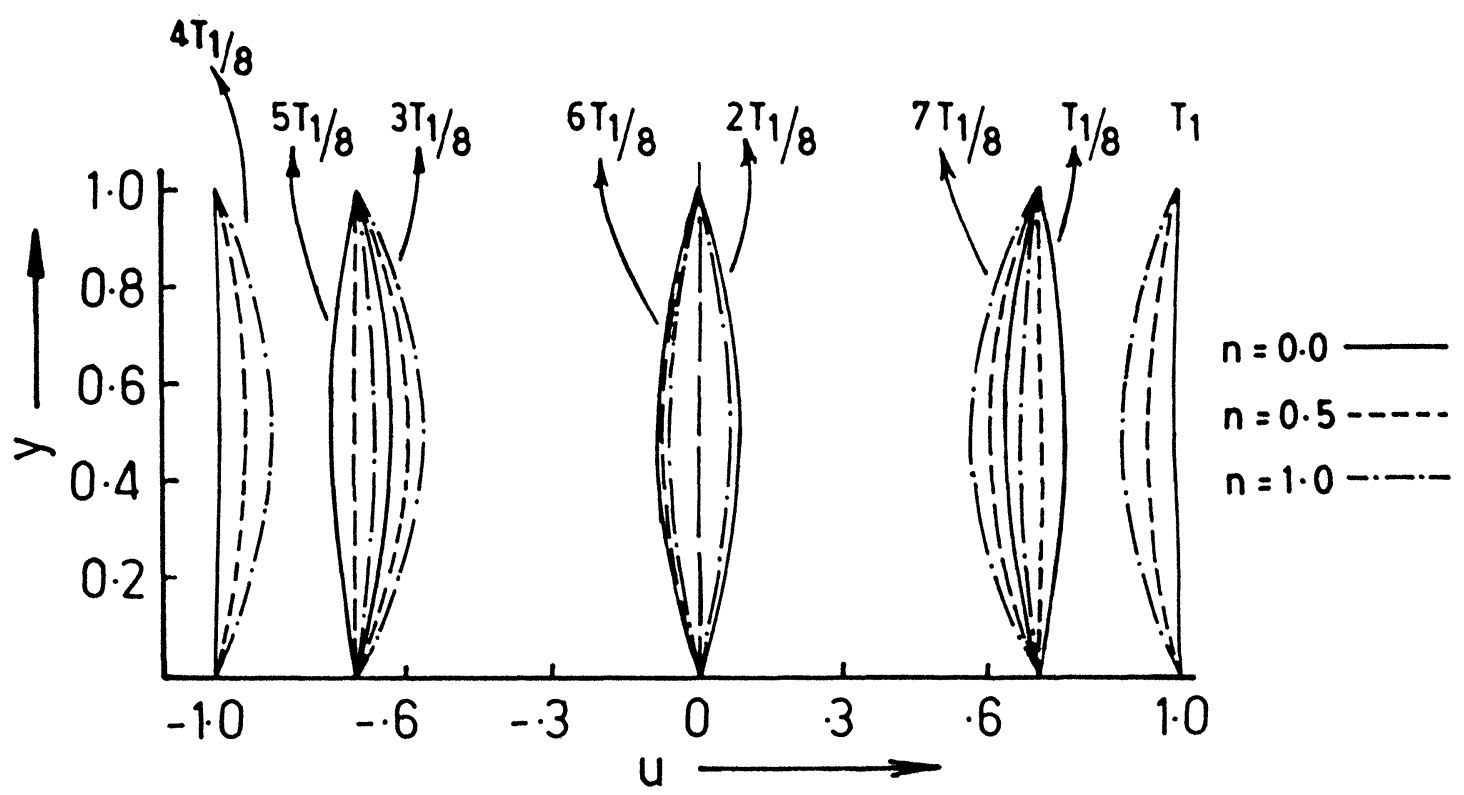

Fig.12. Velocity distribution for the conducting dusty fluid for different values of $n$ when $k=0.1, T=0.1, T_{1}=10.0$ and $\sigma=1.0 \quad\left(T_{1}=T_{2}\right)$

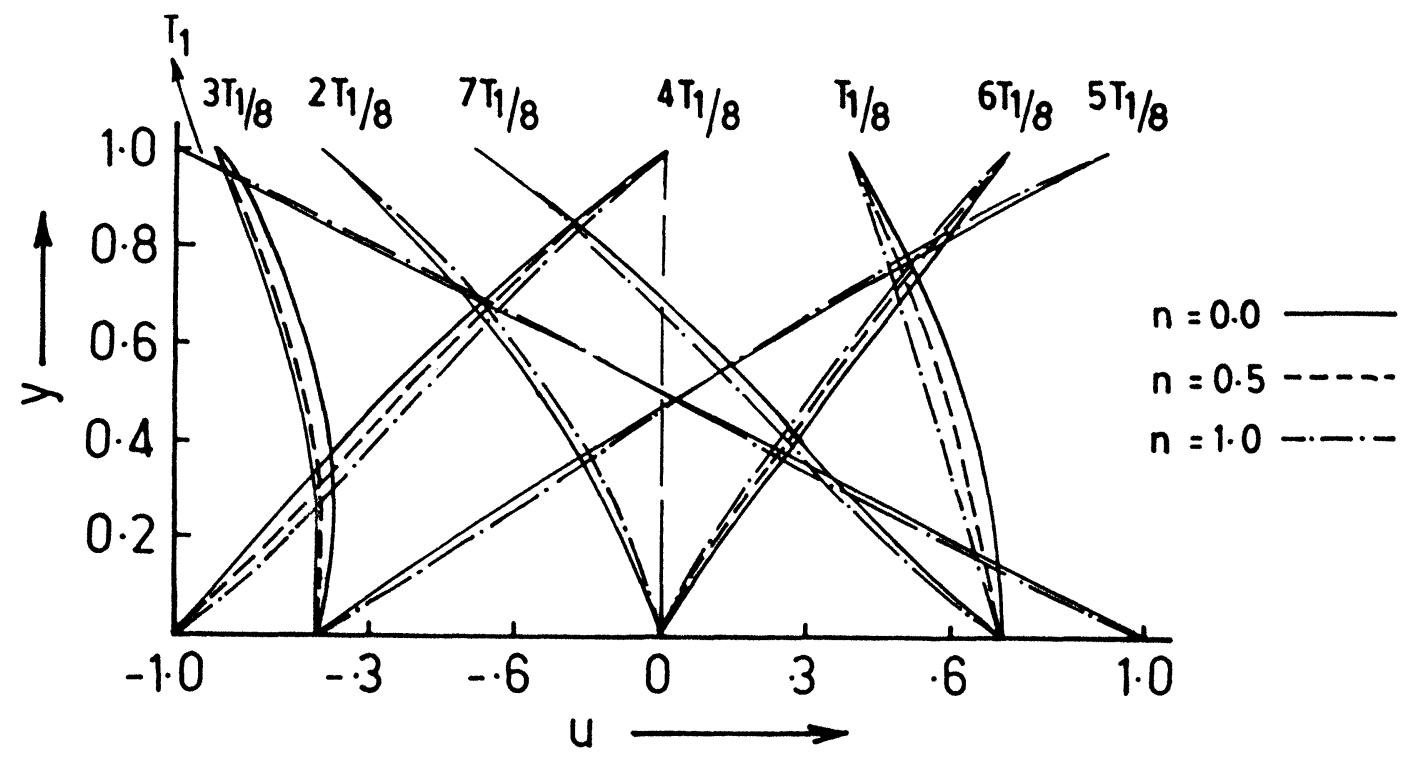

Fig.13. Velocity distribution for the conducting dusty fluid for different values of $n$ when $k=0.1, T=0.1, T_{1}=10.0$ and $\sigma=1.5\left(T_{1}>T_{2}\right)$ 


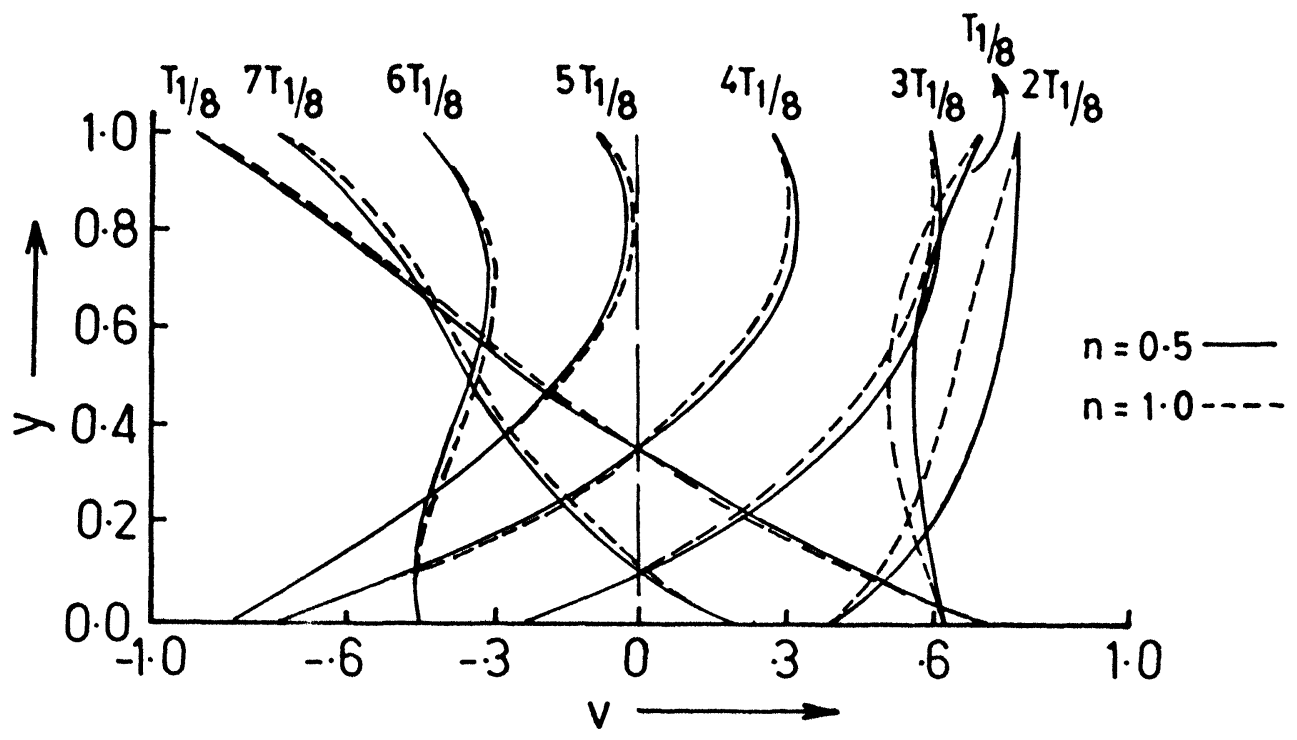

Fig.14. Particle velocity for different values of $n$ when $k=0 \cdot 1, T=0.1$, $T_{1}=1 \cdot 0, \sigma=0.5\left(T_{1}<T_{2}\right)$

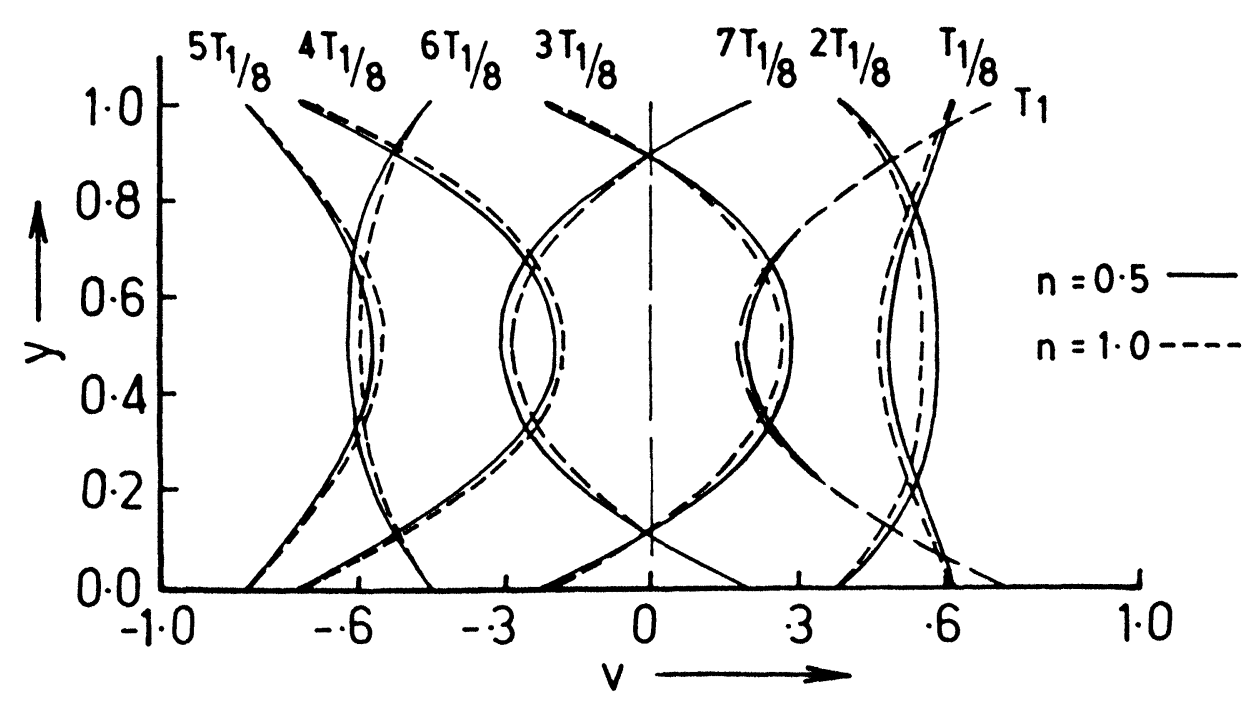

Fig.15. Particle velocity for different values of $n$ when $k=0.1, T=0.1$, $T_{1}=1 \cdot 0, \sigma=1.0\left(T_{1}=T_{2}\right)$ 


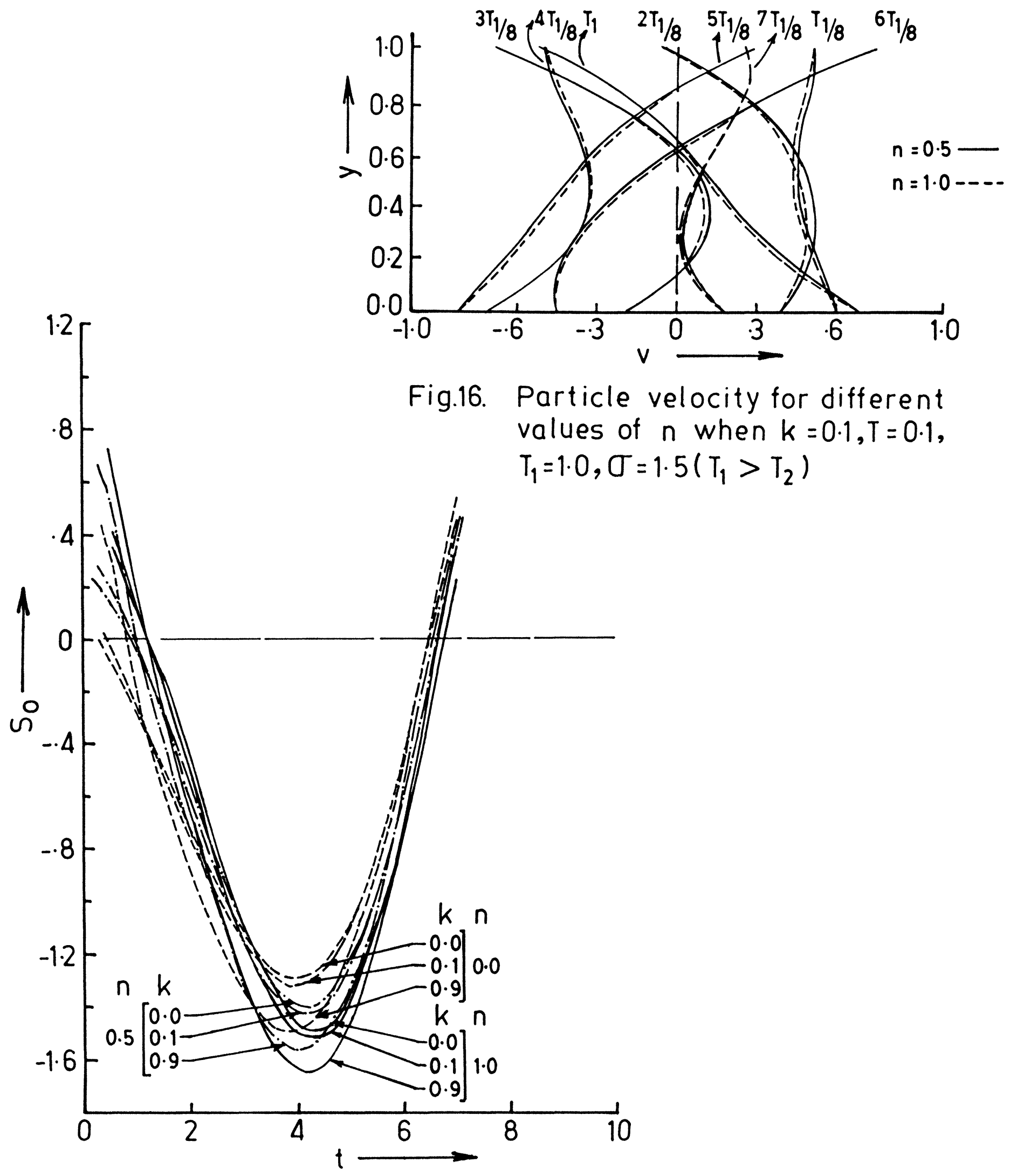

Fig.17. Drag on the plate at $y=0$ when $T=0.3, T_{1}=10.0$ and $\sigma=0.5\left(T_{1}<T_{2}\right)$ 


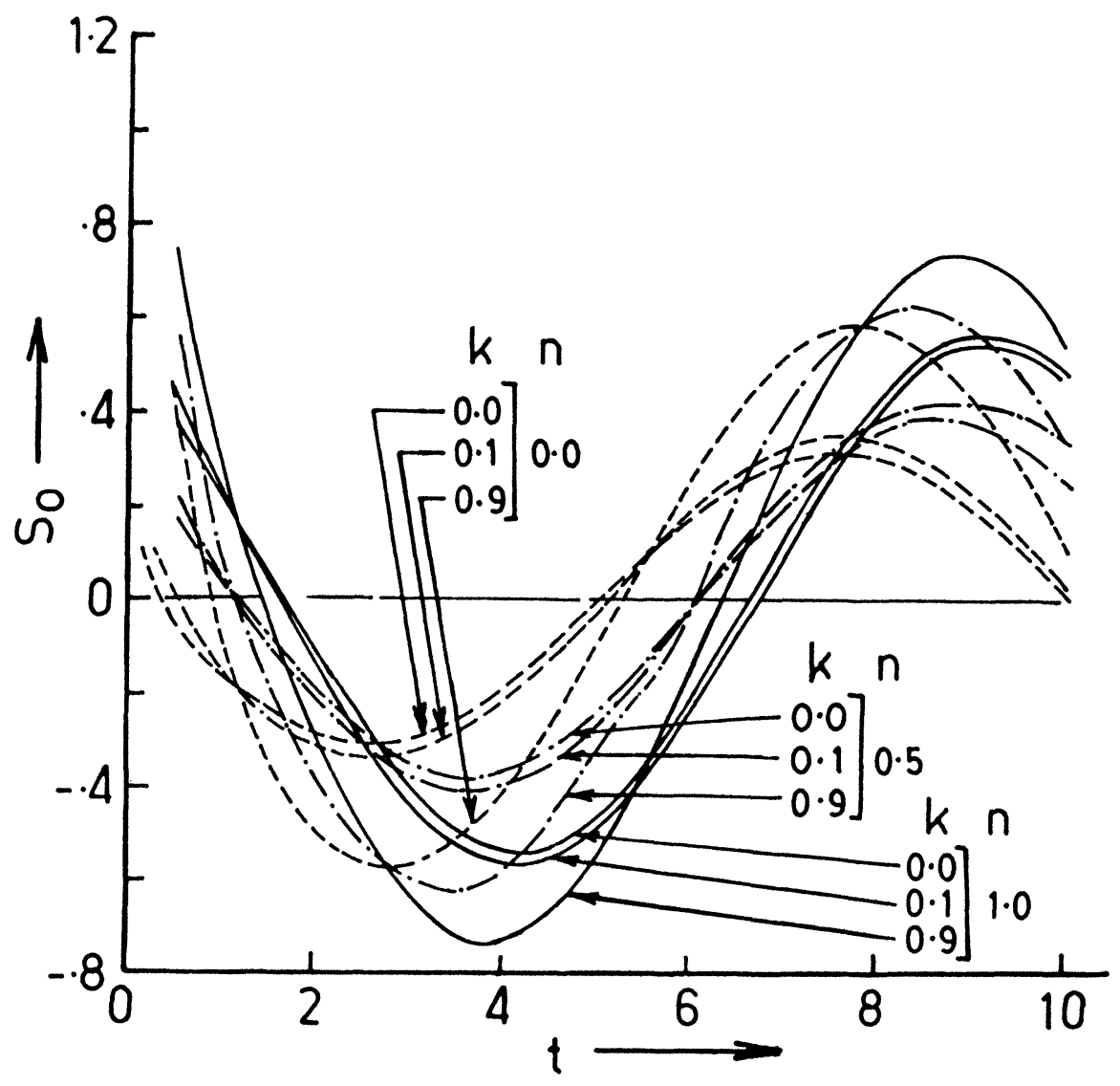

Fig.18. Drag on the plate at $y=0$ when $T=0.3$, $T_{1}=10.0$ and $\sigma=1\left(T_{1}=T_{2}\right)$

Regarding the effect of the magnetic field on the drag, we observe that the drag on the plate increases and decreases with the magnetic field as time advances. In other words, the effect of the magnetic field oscillates with the oscillation of the drag on the plate.

ACKNOWLEDGEMENT. This research is partially supported by the University of Central Florida. 


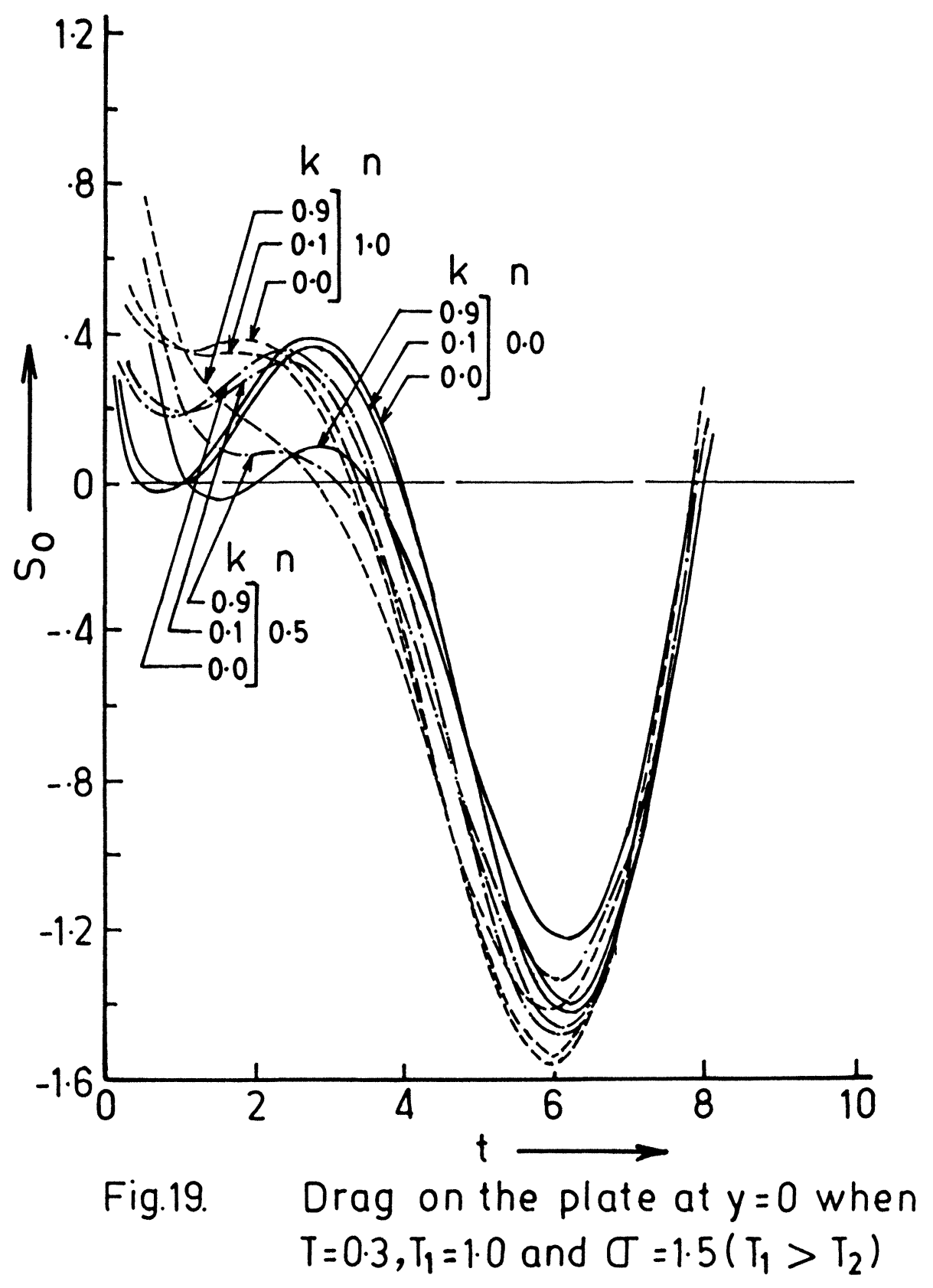




\section{REFERENCES}

[1] P. G. Saffman, On the Stability Laminar Flow of a Dusty Gas. J. Fluid Mech. 13 (1962) 120-128.

[2] A. K. Ghosh and L. Debnath. Hydromagnetic Stokes Flow in a Rotating Fluid with Suspended Small Particles, Appl. Sci. Res. 43 (1986) 165-192.

[3] T. Myint-U and L. Debnath. Partial Differential Equations for Scientists and Engineers, Third Edition, North Holland, 1987.

[4] P. Mitra and P. Bhattacharyya. On the Hydromagnetic Flow of a Dusty Fluid Between Two Parallel Plates, One being Stationary and the Other Oscillating. J. Phy. Soc. Japan, 50 (3) (1981) 995-1001.

[5] P. Mitra and P. Bhattacharyya. Unsteady Hydromagnetic Laminar Flow of a Conducting Dusty Fluid Between Two Parallel Plates Started Impulsively from Rest., Acta Mech. 39 (1981) 171-182.

[6] M. M. Stanisc, B. H. Fetz, H. P. Mickelsen, Jr. and F. M. Czumak. On the Flow of a Hydromagnetic Fluid Between Two Oscillating Flat Plates. J. Aerospace Sci. 29 (1962) 116-117. 


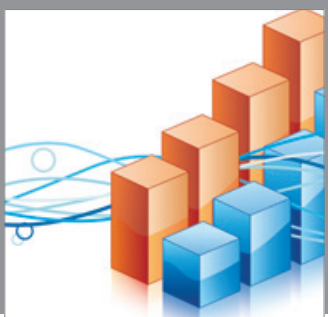

Advances in

Operations Research

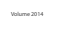

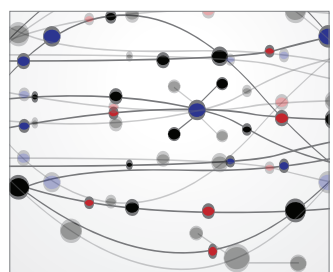

\section{The Scientific} World Journal
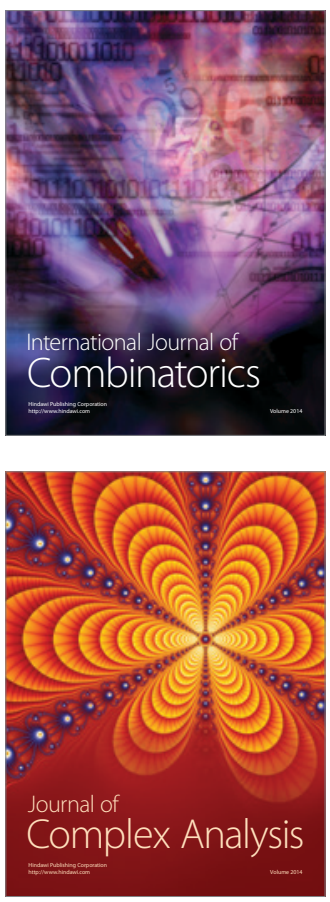

International Journal of

Mathematics and

Mathematical

Sciences
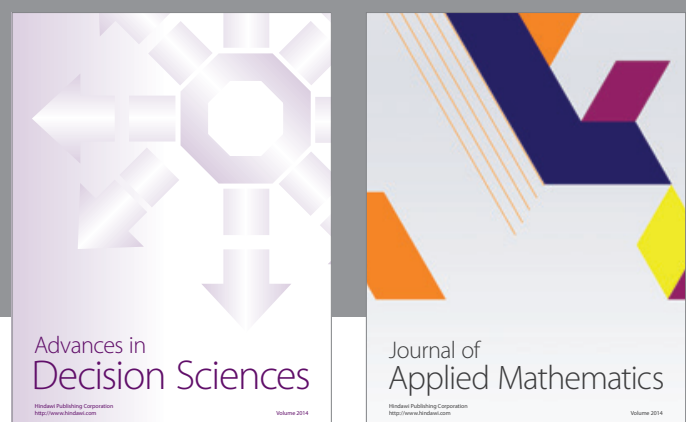

Journal of

Applied Mathematics
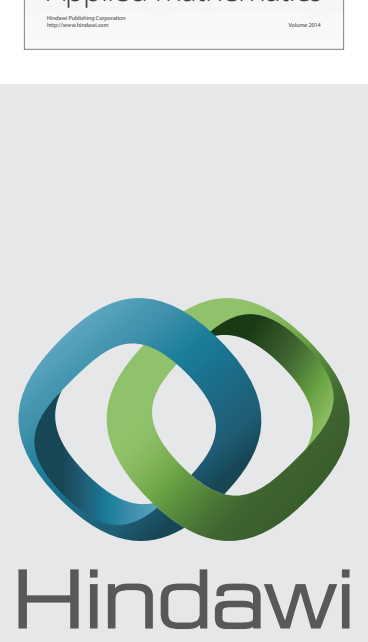

Submit your manuscripts at http://www.hindawi.com
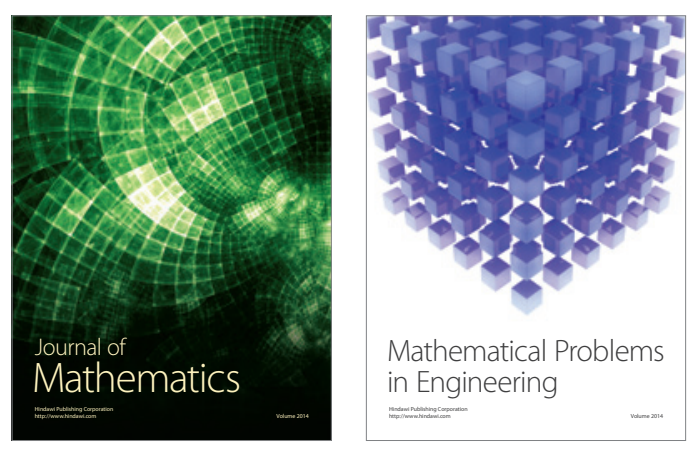

Mathematical Problems in Engineering
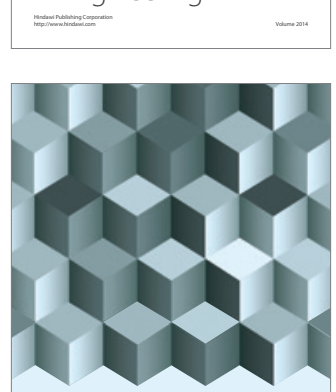

Journal of

Function Spaces
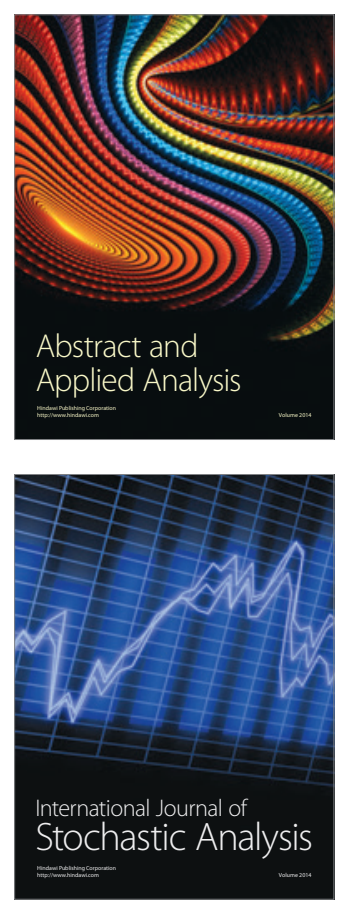

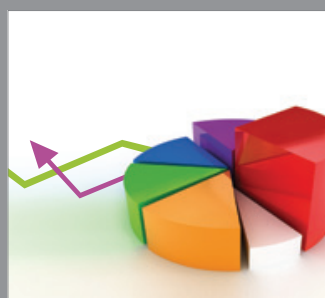

ournal of

Probability and Statistics

Promensencen
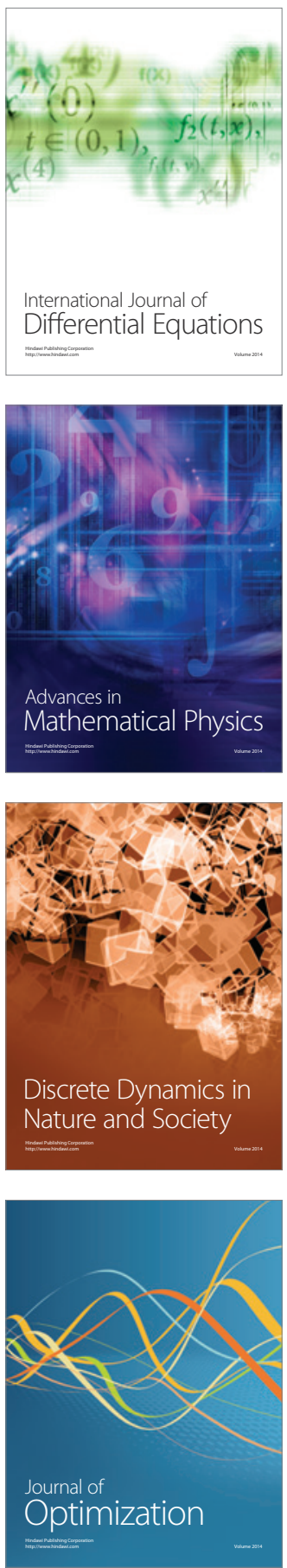